\title{
UTILIZAÇÃO DA ANÁLISE COMPUTADORIZADA DE PLÂNTULAS NA AVALIAÇÃO DO POTENCIAL FISIOLÓGICO DE SEMENTES DE AMENDOIM TRATADAS COM FUNGICIDA E INSETICIDA ${ }^{1}$
}

\author{
JOSÉ LUÍS DE MARCHI², SILVIO MOURE CICERO ${ }^{2}$,FRANCISCO GUILHIEN GOMES JUNIOR ${ }^{4}$
}

\begin{abstract}
RESUMO - As sementes de amendoim são consideradas de difícil produção, pois apresentam frequentemente índices insatisfatórios de germinação e de vigor devido a muitos fatores, dentre eles, problemas associados à ocorrência de fungos e à alta sensibilidade às injúrias mecânicas. Assim, o tratamento das sementes com fungicida, juntamente com inseticidas, visando o controle de pragas iniciais na cultura, é recomendável. Na avaliação do potencial fisiológico de sementes de amendoim, além dos métodos rotineiramente utilizados, a análise computadorizada de plântulas tem grande potencial, pois é método rápido, preciso e sem erros de interpretação humana. $\mathrm{O}$ presente trabalho teve como objetivo avaliar a qualidade fisiológica de sementes de amendoim tratadas com fungicida ou inseticida, bem como a associação de ambos, utilizando-se a técnica de análise computadorizada de plântulas. Sementes do cultivar IAC 886 não tratadas e tratadas com o fungicida Maxim XL (Fludioxonil + Metalaxyl), com o inseticida Cruiser 350 FS (Tiametoxam) e com a mistura de ambos os produtos foram submetidas aos períodos de armazenamento de $0,15,30,45,60,90$ e 180 dias $\left(20{ }^{\circ} \mathrm{C}, 50 \%\right.$ UR do ar). Após cada período de armazenamento, as sementes foram submetidas à avaliação do grau de umidade, germinação, envelhecimento acelerado, emergência de plântulas em campo, condutividade elétrica e sanidade, além da análise computadorizada de plântulas. Concluiu-se que a análise computadorizada de plântulas apresenta eficiência equivalente aos testes de envelhecimento acelerado e de emergência de plântulas em campo na identificação de diferenças entre tratamentos com ausência ou presença de fungicida e de fungicida associado a inseticida nas sementes de amendoim.
\end{abstract}

Termos para indexação: Arachis hypogea, análise de imagens, germinação, vigor

\author{
USING COMPUTERIZED ANALYSIS OF SEEDLINGS TO EVALUATE THE \\ PHYSIOLOGICAL POTENTIAL OF PEANUT SEEDS TREATED WITH FUNGICIDE AND \\ INSETICIDE
}

\begin{abstract}
Peanut seeds are considered difficult to produce because they often show unsatisfactory levels of germination and vigor due to many factors, including problems associated with fungi and a high sensitivity to mechanical injuries. In addition to treating peanut seeds with a fungicide, treatment with insecticides to control soil insect pests in the early growth stages is recommended. Besides using the routine tests for evaluating the physiological potential of peanut seeds, computer analysis of seedlings has great potential because it is accurate, quick and without any human interpretation errors. This study aimed to evaluate the physiological potential and sanitary quality of peanut seeds treated with a fungicide, an insecticide, and a combination of both, using computerized analysis of seedlings. Seeds of the cultivar IAC 886, untreated and treated with the fungicide Maxim XL (fludioxonil + metalaxyl), the insecticide Cruiser 350 FS (thiamethoxam) and with a mixture of both products, were stored for periods of $0,15,30,45$, 60,90 and 180 days $\left(20^{\circ} \mathrm{C}, 50 \%\right.$ air $\left.\mathrm{RH}\right)$. After each storage period, seeds were evaluated for
\end{abstract}

${ }^{1}$ Submetido em 23/07/10. Aceito para publicação em 26/03/11. Parte da Tese de Doutorado do primeiro autor apresentado a USP/ESALQ.

${ }^{2}$ Eng. Agr., pós-graduando do Departamento de Produção Vegetal, USP/ ESALQ, jlmarchi@terra.com.br
${ }^{3}$ Eng. Agr., Dr., Professor Titular, Departamento de Produção Vegetal, USP/ESALQ, bolsista de produtividade em pesquisa do CNPq, Caixa Postal 9, 13418-900. Piracicaba/SP, smcicero@carpa.ciagri.usp.br.

${ }^{4}$ Eng. Agr., Dr, Departamento de Produção Vegetal, USP/ESALQ. 
moisture content, germination, accelerated aging, seedling emergence in the field, electrical conductivity and seed pathology, as well as a computer analysis of the seedlings. It was concluded that computer analysis of peanut seedlings has an efficiency which is equivalent to tests on seeds for accelerated aging and seedling emergence in the field for identifying treatment differences in the absence or presence of a fungicide and a fungicide associated with an insecticide.

Index terms: Arachis hypogea, image analysis, germination, vigor

\section{INTRODUÇÃO}

O amendoim (Arachis hypogaea L.) destaca-se entre as principais oleaginosas cultivadas no Brasil e no mundo. Dentre os produtos derivados das sementes desta leguminosa destaca-se o óleo, que, além de conter alto teor de vitamina $\mathrm{E}$, possui quantidades consideráveis de vitaminas B1 e B2.

No contexto da produção de sementes de grandes culturas, as de amendoim estão entre aquelas que são consideradas de difícil produção, pois apresentam, frequentemente, porcentuais insatisfatórios de germinação (abaixo de $70 \%$ ) e baixo vigor e serem constantemente sujeitas à infecção por microrganismos, em especial, fungos. Além disso, a semente de amendoim é muito sensível às injúrias mecânicas, devido ao tegumento fino e frágil, cotilédones volumosos e quebradiços e a extremidade da radícula em posição próxima à superfície basal dos cotilédones; tais características proporcionam alta vulnerabilidade na operação de descasque, ocasionando problemas também nas operações de processamento, beneficiamento, transporte e semeadura (Delouche e Baskin, 1973; Gelmond, 1971; Sader et al., 1991). Assim, condições fisiológicas e sanitárias insatisfatórias associadas às injúrias mecânicas facilitam a infecção dos cotilédones e do eixo embrionário por microorganismos presentes nas sementes ou no solo (Aspergillus spp., Fusarium spp., Rhizoctonia spp., Rhizopus spp., Sclerotium spp., dentre outros), provocando a morte das sementes ou o tombamento das plântulas e, consequentemente, a diminuição da população de plantas (Machado, 1987; Moraes, 1987; Menten, 1991). Dessa maneira, o tratamento da semente com fungicida contribui para a manutenção da germinação constituindo prática recomendada (Moraes, 1987; Maeda et al. 1995). Sua vantagem visando à redução dos prejuízos causados por patógenos na germinação e na emergência de plântulas tem sido demonstrada por diversos pesquisadores (Tosello et al., 1970; Backman e Hammond, 1976; Lago et al.,
1976; Tella et al., 1976; Maeda et al., 1995 e RandallSchadel et al., 2001). Porém, o momento de sua aplicação não proporcionou diferenças significativas nos índices de germinação (Usberti, 1983; Angelini e Martins, 1986; Prete e Cicero, 1987).

Além do tratamento fungicida, o tratamento das sementes com inseticidas visando o controle de pragas iniciais da cultura é de grande importância, destacandose o uso racional de produtos eficazes e de prolongada ação residual, como os inseticidas fosforados. Uma das preocupações na utilização de inseticidas fosforados no tratamento de sementes é o seu possível efeito nocivo sobre o potencial fisiológico. Fadigas Júnior e Suplicy Filho (1961) não observaram danos à emergência de plântulas com a utilização de inseticidas fosforados sistêmicos no tratamento de sementes de amendoim. Por outro lado, vários trabalhos de pesquisa evidenciaram efeito prejudicial do produto, quer seja sobre a emergência de plântulas (Lago e Toledo, 1970), ou ocasionando fitotoxicidade ao longo do armazenamento de sementes de amendoim (Scarpellini e Nakamura, 2002) e de milho (Gotardo et al., 2001). Pandey e Singh (2006) trataram sementes de amendoim com os inseticidas Chlorpyrifos 20 EC e Quinalphos 25 EC, bem como realizaram a aplicação dos produtos diretamente no solo e não verificaram efeitos negativos para a germinação das sementes; contudo, observaram mudanças na atividade de enzimas da solução do solo, as quais podem afetar negativamente a disponibilidade de nutrientes para a planta e ocasionar quedas na produtividade, quando utilizados por longo período de tempo e em doses inadequadas.

Os testes de vigor tradicionalmente utilizados para a avaliação da qualidade de sementes são amplamente divulgados e aceitos pela indústria de sementes como instrumento para tomada de decisões (AOSA, 1983, Delouche, 1986; Krzyzanowsky e Miranda, 1990). Dentro desse contexto, tem sido presenciada uma evolução favorável à utilização de técnicas computadorizadas mais sensíveis para a captação e também para o processamento e 
extração de informações úteis para a indústria de sementes, definindo-se uma amplificação de sensibilidade por vias digitais (Teixeira et al., 2003). Assim, pesquisadores da Universidade Estadual de Ohio, EUA (Sako et al., 2001), elaboraram um sistema automatizado para avaliação do vigor de sementes de alface, o SVIS ${ }^{\circledR}$ ("Seed Vigor Imaging System").

O procedimento para a avaliação do vigor de sementes por meio deste sistema automatizado é simples e os resultados para uma amostra de 50 sementes podem ser obtidos dentro de dois a três minutos (Gomes Junior et al., 2009). Outras vantagens deste sistema incluem a eliminação do erro humano, aumentando a confiabilidade dos dados para fins de comparação, a possibilidade de arquivamento das imagens para análise posterior e o baixo custo, que, segundo Gomes Junior et al. (2009), é equivalente ao custo do teste de germinação.

Sako et al. (2001) demonstraram que o processamento digital de imagens pode ser utilizado com sucesso em plântulas de alface e de soja, na avaliação do vigor das sementes. Da mesma forma, Marcos Filho et al. (2006) comparando os métodos tradicionais para a avaliação do vigor de sementes de melão, com este sistema automatizado de análise de imagens, constataram seu grande potencial como alternativa para a avaliação do vigor das sementes. Após algumas adaptações, também tem sido eficiente na avaliação do potencial fisiológico de sementes de outras espécies, como milho (Hoffmaster et al., 2005; Otoni e McDonald, 2005; Gomes Junior et al., 2009 e feijão (Gomes Junior e Chama, 2008). Estas evidências indicam que este programa representa uma técnica promissora para avaliação do vigor de sementes de outras espécies em diferentes finalidades na área de Tecnologia de Sementes. Neste contexto, o uso desse sistema automatizado pode ser eficiente na identificação de diferenças entre lotes de sementes submetidos ou não ao tratamento químico.

Nãoforamconstatadosnaliteraturadisponível trabalhos de pesquisa envolvendo a análise computadorizada de plântulas na avaliação da qualidade de sementes de amendoim. Assim, o presente trabalho teve como objetivo avaliar o potencial fisiológico de sementes de amendoim tratadas com fungicida e inseticida, bem como a associação de ambos, utilizando-se a referida técnica.

\section{MATERIAL E MÉTODOS}

O experimento foi conduzido em 2008, nos Laboratórios de Análise de Sementes e de Análise de Imagens do Departamento de Produção Vegetal (LPV) e no Laboratório de Patologia de Sementes do Departamento de Fitopatologia e Nematologia (LFN) da Escola Superior de Agricultura "Luiz de Queiroz" (ESALQ), Universidade de São Paulo (USP), em Piracicaba, SP. Foram utilizadas sementes do cultivar IAC 886, as quais foram submetidas aos tratamentos descritos na Tabela 1 .

TABELA 1. Nome comercial, nome técnico e dosagens dos produtos químicos utilizados nos tratamentos das sementes de amendoim, cultivar IAC 886.

\begin{tabular}{cccc}
\hline Tratamento & Nome Comercial & Nome Técnico & Dosagem $\left(\mathrm{mL} .1000 \mathrm{~kg}^{-1}\right)$ \\
\hline 1 & Testemunha & & \\
2 & (sem tratamento) & Fludioxonil + Metalaxyl & 100 \\
3 & Maxim XL & Tiametoxam & 150 \\
4 & Cruiser 350 FS & (Fludioxonil + Metalaxyl) + & $100+150$ \\
\hline
\end{tabular}

Após a obtenção dos tratamentos, as sementes foram acondicionadas em embalagens de papel kraft e armazenadas em câmara seca $\left(20^{\circ} \mathrm{C}\right.$ e $50-60 \%$ de umidade relativa do ar).

A qualidade fisiológica e sanitária das sementes foi avaliada imediatamente após a obtenção dos tratamentos, com início a 0 dias de armazenamento, e durante os períodos correspondentes a 15, 30, 45, 60, 90 e 180 dias de armazenamento. As amostras, correspondentes a cada tratamento foram submetidas às avaliações descritas a seguir.

Determinação do grau de umidade: efetuada em estufa a $105 \pm 3{ }^{\circ} \mathrm{C}$, durante 24 horas, conforme as Regras para Análise de Sementes (Brasil, 1992), com exceção do número de repetições, que foram quatro, com dez sementes cada. Os resultados foram expressos em porcentagem (base úmida).

Teste de germinação: para cada tratamento, foram 
semeadas quatro repetições de 50 sementes em caixas plásticas de 47 × $30 \times 11 \mathrm{~cm}$. As sementes foram semeadas em areia lavada e esterilizada com brometo de metila. A avaliação final das plântulas normais foi feita aos 15 dias após a semeadura, seguindo-se os critérios das Regras para Análise de Sementes (Brasil, 1992). O substrato foi irrigado com água até atingir $60 \%$ da sua capacidade de retenção. As caixas permaneceram em condições de ambiente não controlado, sendo os resultados expressos em porcentagem.

Teste de envelhecimento acelerado: quatro repetições de 50 sementes para cada tratamento foram distribuídas sobre uma tela de alumínio, fixada em caixas de plástico do tipo gerbox, contendo $40 \mathrm{~mL}$ de água destilada, as quais foram acondicionadas em câmara de envelhecimento por 24 horas a $42 \pm 1{ }^{\circ} \mathrm{C}$ e UR do ar de aproximadamente $100 \%$. Após esse período, as sementes foram submetidas ao teste de germinação, sendo a avaliação realizada no quinto dia após a instalação (Marcos Filho et al., 1987). O teor de água das sementes foi determinado antes e após o período de envelhecimento.

Emergência de plântulas em campo: foi conduzida com quatro repetições de 100 sementes para cada tratamento. A profundidade de semeadura foi de $3-4 \mathrm{~cm}$ em sulco de $4 \mathrm{~m}$ de comprimento. O solo foi mantido com umidade suficiente para promover a emergência das plântulas por meio de irrigação. A avaliação foi realizada em contagem única, aos 21 dias após a semeadura, computando-se o número de plântulas emersas. O resultado foi expresso em porcentagem.

Condutividade elétrica: foi realizada conforme a recomendação do Comitê de Vigor da "Association of Official Seed Analysts" (AOSA, 1983). Foram utilizadas quatro repetições de 25 sementes fisicamente puras por tratamento. Após a pesagem, as sementes de cada repetição foram transferidas para copos plásticos contendo $75 \mathrm{~mL}$ de água destilada e mantidos em $20{ }^{\circ} \mathrm{C}$, durante 24 horas. Decorrido esse período a condutividade elétrica da solução foi determinada por condutivímetro DIGIMED ${ }^{\circledR}$, modelo DM-31, sendo os resultados expressos em micromhos por centímetro por grama de sementes.

Teste de sanidade: foi realizado com base em metodologia descrita por Luca Filho (1987), empregandose duas repetições de 100 sementes de cada tratamento, que foram incubadas em placas de Petri (10 sementes por placa) contendo três folhas de papel de filtro umedecidas em água destilada. As placas contendo as sementes foram incubadas por sete dias à temperatura de $20{ }^{\circ} \mathrm{C}$, sob luz branca fluorescente alternada (12 horas de luz por 12 horas de escuro). Após o período de incubação, foram realizadas a avaliação e identificação dos patógenos sob microscópio estereoscópico, analisando-se individualmente as sementes. Os resultados foram expressos em porcentagem de incidência de cada patógeno nas sementes.

Análise computadorizada de plântulas: realizada com quatro repetições de 50 sementes para cada tratamento. As sementes foram distribuídas em grupos de 25 (cinco fileiras de cinco sementes) em folhas de papel germitest, para germinação, umedecidas com água equivalente a 2,5 vezes a sua massa. Os rolos foram colocados em germinador e mantidos em $25^{\circ} \mathrm{C}$. A aquisição das imagens foi realizada no quinto dia após a instalação do teste.

A aquisição das imagens das plântulas foi realizada pelo sistema de escaneamento computadorizado e programa computacional de processamento de imagens descritos por Hoffmaster et al. (2003). Assim, as imagens das plântulas normais ou anormais ou de sementes mortas foram obtidas por meio de um escâner HP Scanjet 2400 com área de escaneamento de $30 \mathrm{~cm}$ x $21 \mathrm{~cm}$, instalado em posição invertida, no interior de uma caixa de alumínio com $60 \times 50$ × $12 \mathrm{~cm}$, ajustado na resolução de 100 dpi e acoplado a um computador Core 2 Duo (2GB de memória RAM e HD de 160GB). As imagens foram processadas, sendo gerados os índices de vigor (IV), de crescimento (IC) e de uniformidade de crescimento (IU). O índice de vigor é resultante da combinação entre os índices de crescimento e de uniformidade, que podem variar de um valor mínimo de zero a um máximo de 1000 . Os dados foram obtidos estabelecendo como $4,0 \mathrm{~cm}$ o tamanho máximo de plântula. Para a obtenção do índice de vigor foi utilizada a seguinte composição: IV70/30 $=\{(0,7 \times$ IC $)+(0,3$ x IU $)\}$. Foram realizados, também, pequenos ajustes, para reconhecer e marcar corretamente o ponto de ligação entre a raiz primária e a parte aérea das plântulas normais.

O delineamento experimental adotado foi o inteiramente casualizado, no esquema fatorial $4 \times 7$. Os dados provenientes dos testes de germinação, envelhecimento acelerado e emergência de plântulas em campo e de sanidade, foram transformados em arc sen $\sqrt{x / 100}$. As médias foram comparadas pelo teste de Tukey noo nível de $5 \%$ de probabilidade.

\section{RESULTADOS E DISCUSSÃO}

Os resultados do grau de umidade, de germinação, de envelhecimento acelerado, de emergência de 
plântulas em campo e de condutividade elétrica das sementes, submetidas ou não ao tratamento químico e armazenadas por diferentes períodos estão apresentados na Tabela 2. Na Tabela 3 são apresentados os porcentuais de incidência de fungos nas sementes e na Tabela 4 os resultados dos índices de crescimento, de uniformidade e de vigor obtidos a partir da análise computadorizada de plântulas.

TABELA 2. Resultados do grau de umidade (U) e dos testes de germinação (TG), de envelhecimento acelerado (EA), de emergência de plântulas em campo (EC) e de condutividade elétrica (CE) das sementes de amendoim, do cultivar IAC 886, após serem submetidas aos tratamentos químicos (TQ) e diferentes períodos de armazenamento.

\begin{tabular}{|c|c|c|c|c|c|c|}
\hline DATQ $^{(1)}$ & $\mathrm{TQ}^{(2)}$ & $\mathrm{U}$ & $\mathrm{T} \mathrm{G}$ & EA & $\mathrm{EC}$ & \multirow{2}{*}{$\begin{array}{c}\text { CE } \\
\left(\mu{\mathrm{S} . c m^{-1}}^{\prime} \cdot \mathrm{g}^{1}\right)\end{array}$} \\
\hline & & \multicolumn{4}{|c|}{ 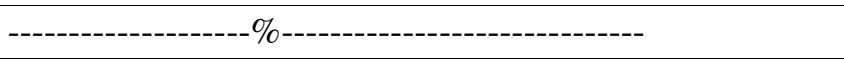 } & \\
\hline \multirow{4}{*}{0} & $\mathrm{~T}$ & $5,4 \mathrm{Aa}$ & $58 \mathrm{AB} * \mathrm{a} * *$ & $19 \mathrm{CDb}$ & $44 \mathrm{Cb}$ & $27,8 \mathrm{Aa}$ \\
\hline & $\mathrm{F}$ & $5,5 \mathrm{Aa}$ & $67 \mathrm{Aa}$ & $39 \mathrm{Ba}$ & $67 \mathrm{Aa}$ & $33,0 \mathrm{Aa}$ \\
\hline & FI & $5,1 \mathrm{Aa}$ & $65 \mathrm{Aa}$ & $35 \mathrm{Ba}$ & $63 \mathrm{Aa}$ & $32,8 \mathrm{Aa}$ \\
\hline & I & $5,3 \mathrm{Aa}$ & $58 \mathrm{ABa}$ & $18 \mathrm{Db}$ & 34Dc & $32,5 \mathrm{Aa}$ \\
\hline \multirow{4}{*}{15} & $\mathrm{~T}$ & $5,1 \mathrm{Aa}$ & $52 \mathrm{Bb}$ & $14 \mathrm{Db}$ & $42 \mathrm{Cb}$ & $34,3 \mathrm{Aa}$ \\
\hline & $\mathrm{F}$ & $5,5 \mathrm{Aa}$ & $65 \mathrm{Aa}$ & $53 \mathrm{Aa}$ & $57 \mathrm{Ba}$ & $33,4 \mathrm{Aa}$ \\
\hline & FI & $5,5 \mathrm{Aa}$ & $52 \mathrm{Bb}$ & $48 \mathrm{ABa}$ & $47 \mathrm{Cab}$ & $34,1 \mathrm{Aa}$ \\
\hline & I & $5,4 \mathrm{Aa}$ & $51 \mathrm{Bb}$ & $13 \mathrm{Db}$ & $35 \mathrm{Dc}$ & $36,9 \mathrm{Bb}$ \\
\hline \multirow{4}{*}{30} & $\mathrm{~T}$ & $5,5 \mathrm{Aa}$ & $52 \mathrm{Ab}$ & $11 \mathrm{Dc}$ & $42 \mathrm{Cb}$ & $37,0 \mathrm{Ba}$ \\
\hline & $\mathrm{F}$ & $5,9 \mathrm{Aa}$ & $62 \mathrm{Aa}$ & $37 \mathrm{Ba}$ & 59Aba & $41,4 \mathrm{Bb}$ \\
\hline & FI & $5,4 \mathrm{Aa}$ & $60 \mathrm{ABa}$ & $21 \mathrm{Cb}$ & $56 \mathrm{Ba}$ & $40,3 \mathrm{Bb}$ \\
\hline & I & $5,3 \mathrm{Aa}$ & $51 \mathrm{Bb}$ & $8 \mathrm{Dc}$ & $35 \mathrm{Db}$ & $44,0 \mathrm{Cb}$ \\
\hline \multirow{4}{*}{45} & $\mathrm{~T}$ & $5,5 \mathrm{Aa}$ & $56 \mathrm{ABa}$ & $10 \mathrm{Db}$ & $36 \mathrm{Db}$ & $45,1 \mathrm{Ca}$ \\
\hline & $\mathrm{F}$ & $5,5 \mathrm{Aa}$ & $63 \mathrm{Aa}$ & 30BCa & $53 \mathrm{Ba}$ & $44,0 \mathrm{Ca}$ \\
\hline & FI & $5,6 \mathrm{Aa}$ & $62 \mathrm{Aa}$ & $18 \mathrm{CDb}$ & $53 \mathrm{Ba}$ & $43,3 \mathrm{Ca}$ \\
\hline & I & $5,8 \mathrm{Aa}$ & $45 \mathrm{Bb}$ & $5 \mathrm{~Eb}$ & $27 \mathrm{Ec}$ & $43,6 \mathrm{Ca}$ \\
\hline \multirow{4}{*}{60} & $\mathrm{~T}$ & $5,6 \mathrm{Aa}$ & $33 \mathrm{Cb}$ & $7 \mathrm{Db}$ & $40 \mathrm{Cb}$ & $45,0 \mathrm{Da}$ \\
\hline & $\mathrm{F}$ & $5,4 \mathrm{Aa}$ & $53 \mathrm{Ba}$ & $28 \mathrm{Ca}$ & $60 \mathrm{Aa}$ & $42,7 \mathrm{Ca}$ \\
\hline & FI & $5,4 \mathrm{Aa}$ & $52 \mathrm{Ba}$ & $22 \mathrm{Ca}$ & $43 \mathrm{Cb}$ & $41,9 \mathrm{Ca}$ \\
\hline & I & $5,5 \mathrm{Aa}$ & $27 \mathrm{Cb}$ & $3 \mathrm{~Eb}$ & 30Dc & $42,6 \mathrm{Ca}$ \\
\hline \multirow{4}{*}{90} & $\mathrm{~T}$ & $5,5 \mathrm{Aa}$ & $31 \mathrm{Cb}$ & $5 \mathrm{~Eb}$ & 32Dc & $48,2 \mathrm{CDa}$ \\
\hline & $\mathrm{F}$ & $5,0 \mathrm{Aa}$ & $52 \mathrm{Ba}$ & $24 \mathrm{Ca}$ & $54 \mathrm{Ba}$ & $50,8 \mathrm{Da}$ \\
\hline & FI & $5,5 \mathrm{Aa}$ & $38 \mathrm{Bb}$ & $24 \mathrm{Ca}$ & $41 \mathrm{Cb}$ & $53,7 \mathrm{Da}$ \\
\hline & I & $5,2 \mathrm{Aa}$ & $21 \mathrm{Dc}$ & $1 \mathrm{~Eb}$ & $26 \mathrm{Ec}$ & $47,4 \mathrm{CDa}$ \\
\hline \multirow{4}{*}{180} & $\mathrm{~T}$ & $5,1 \mathrm{Aa}$ & $19 \mathrm{Db}$ & $2 \mathrm{~Eb}$ & $24 \mathrm{Ec}$ & $64,5 \mathrm{Da}$ \\
\hline & $\mathrm{F}$ & $5,3 \mathrm{Aa}$ & $35 \mathrm{Ca}$ & $16 \mathrm{Da}$ & $52 \mathrm{Ba}$ & $72,3 \mathrm{Db}$ \\
\hline & FI & $4,5 \mathrm{Aa}$ & $30 \mathrm{Ca}$ & $12 \mathrm{Da}$ & $36 \mathrm{Cb}$ & $67,9 \mathrm{Da}$ \\
\hline & I & $5,5 \mathrm{Aa}$ & $16 \mathrm{Db}$ & $0 \mathrm{~Eb}$ & $24 \mathrm{Ec}$ & $70,3 \mathrm{Db}$ \\
\hline $\mathrm{CV}(\%)$ & & 8,74 & 6,58 & 15,02 & 6,34 & 9,20 \\
\hline
\end{tabular}

${ }^{1}$ Dias Após o Tratamento Químico (DATQ). ${ }^{2} \mathrm{~T}=$ Testemunha; F= Fludioxonil + metalaxyl; FI= Fludioxonil+metalaxyl + Tiametoxam; I= Tiametoxam

*Valores seguidos pela mesma letra maiúscula, em cada coluna, não diferem entre si pelo teste de Tukey, aos $5 \%$ de probabilidade. **Valores seguidos pela mesma letra minúscula, em cada coluna, dentro dos diferentes períodos correspondentes aos dias após o tratamento químico, não diferem entre si pelo teste de Tukey, aos $5 \%$ de probabilidade 
TABELA 3. Incidência de fungos em sementes de amendoim, cultivar IAC 886, submetidas ao tratamento químico (TQ) e armazenadas por diferentes períodos.

\begin{tabular}{|c|c|c|c|c|}
\hline \multirow{2}{*}{$\operatorname{DATQ}^{(1)}$} & \multirow{2}{*}{$\mathrm{TQ}^{(2)}$} & Aspergillus spp. & Rhizopus spp. & Penicillum spp. \\
\hline & & \multicolumn{3}{|c|}{ 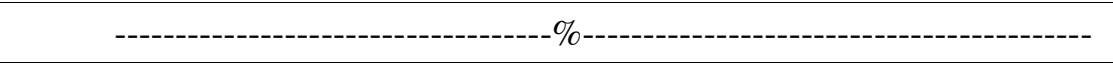 } \\
\hline \multirow{4}{*}{0} & $\mathrm{~T}$ & $4 \mathrm{~A} * \mathrm{a} * *$ & $51 \mathrm{Cb}$ & $0 \mathrm{Aa}$ \\
\hline & $\mathrm{F}$ & $0 \mathrm{Aa}$ & $14 \mathrm{Ba}$ & $0 \mathrm{Aa}$ \\
\hline & FI & $2 \mathrm{Aa}$ & $14 \mathrm{Ba}$ & $0 \mathrm{Aa}$ \\
\hline & I & $17 \mathrm{Ab}$ & $50 \mathrm{Cb}$ & OAa \\
\hline \multirow{4}{*}{15} & $\mathrm{~T}$ & $10 \mathrm{Ab}$ & $36 \mathrm{BCb}$ & $1 \mathrm{Aa}$ \\
\hline & $\mathrm{F}$ & $1 \mathrm{Aa}$ & $3 \mathrm{Aa}$ & $0 \mathrm{Aa}$ \\
\hline & FI & $1 \mathrm{Aa}$ & $12 \mathrm{Ba}$ & $0 \mathrm{Aa}$ \\
\hline & I & $22 \mathrm{ABc}$ & $52 \mathrm{Cb}$ & $0 \mathrm{Aa}$ \\
\hline \multirow{4}{*}{30} & $\mathrm{~T}$ & $36 \mathrm{Bb}$ & $63 \mathrm{Cb}$ & $0 \mathrm{Aa}$ \\
\hline & $\mathrm{F}$ & $0 \mathrm{Aa}$ & $12 \mathrm{Ba}$ & $0 \mathrm{Aa}$ \\
\hline & FI & $1 \mathrm{Aa}$ & $17 \mathrm{Ba}$ & $0 \mathrm{Aa}$ \\
\hline & I & $36 \mathrm{Bb}$ & $66 \mathrm{Cb}$ & $0 \mathrm{Aa}$ \\
\hline \multirow{4}{*}{45} & $\mathrm{~T}$ & $31 \mathrm{Bb}$ & $61 \mathrm{Cb}$ & OAa \\
\hline & $\mathrm{F}$ & $0 \mathrm{Aa}$ & $7 \mathrm{ABa}$ & 0Aa \\
\hline & FI & $0 \mathrm{Aa}$ & $19 \mathrm{Ba}$ & $0 \mathrm{Aa}$ \\
\hline & $\mathrm{I}$ & $42 \mathrm{Cb}$ & $78 \mathrm{Db}$ & $0 \mathrm{Aa}$ \\
\hline \multirow{4}{*}{60} & $\mathrm{~T}$ & $30 \mathrm{Bb}$ & $63 \mathrm{Cb}$ & $3 \mathrm{Aa}$ \\
\hline & $\mathrm{F}$ & $4 \mathrm{Aa}$ & $27 \mathrm{Ba}$ & $0 \mathrm{Aa}$ \\
\hline & FI & $4 \mathrm{Aa}$ & $29 \mathrm{Ba}$ & $0 \mathrm{Aa}$ \\
\hline & I & $44 \mathrm{Cb}$ & $86 \mathrm{Dc}$ & $4 \mathrm{Aa}$ \\
\hline \multirow{4}{*}{90} & $\mathrm{~T}$ & $47 \mathrm{Cb}$ & $76 \mathrm{CDb}$ & $3 \mathrm{Aa}$ \\
\hline & $\mathrm{F}$ & $8 \mathrm{Aa}$ & $20 \mathrm{Ba}$ & $4 \mathrm{Aa}$ \\
\hline & FI & $32 \mathrm{Ba}$ & $35 \mathrm{BCa}$ & $0 \mathrm{Aa}$ \\
\hline & I & 63Dc & $81 \mathrm{Db}$ & $4 \mathrm{Aa}$ \\
\hline \multirow{4}{*}{180} & $\mathrm{~T}$ & $57 \mathrm{Dbc}$ & 90Dd & $2 \mathrm{Aa}$ \\
\hline & $\mathrm{F}$ & $16 \mathrm{Aa}$ & $27 \mathrm{Ba}$ & OAa \\
\hline & FI & $32 \mathrm{Bb}$ & $42 \mathrm{BCb}$ & $4 \mathrm{Aa}$ \\
\hline & I & 63Dc & $63 \mathrm{Cc}$ & $4 \mathrm{Aa}$ \\
\hline \multicolumn{2}{|c|}{$\mathrm{CV}(\%)$} & 63,22 & 42,28 & 388,02 \\
\hline
\end{tabular}

${ }^{1}$ Dias Após o Tratamento Químico (DATQ)

${ }^{2} \mathrm{~T}=$ Testemunha; $\mathrm{F}=$ Fludioxonil+metalaxyl; FI= Fludioxonil + metalaxyl + Tiametoxam; $\mathrm{I}=$ Tiametoxa

*Valores seguidos pela mesma letra maiúscula, em cada coluna, não diferem entre si pelo teste de Tukey, aos 5\% de probabilidade

**Valores seguidos pela mesma letra minúscula, em cada coluna, dentro dos diferentes períodos correspondentes aos dias após o tratamento químico, não diferem entre si pelo teste de Tukey, aos $5 \%$ de probabilidade 
TABELA 4. Resultados da avaliação do vigor por meio da análise computadorizada de plântulas: valores médios do índice de crescimento (IC), índice de uniformidade (IU) e índice de vigor (IV) para as sementes de amendoim, cultivar IAC 886, após serem submetidas aos tratamentos químicos (TQ) e diferentes períodos de armazenamento.

\begin{tabular}{|c|c|c|c|c|}
\hline DATQ $^{(1)}$ & $\mathrm{TQ}^{(2)}$ & IC & IU & IV \\
\hline \multirow{4}{*}{0} & $\mathrm{~T}$ & $432 \mathrm{C} * \mathrm{~b} * *$ & 761Aa & $530 \mathrm{Cb}$ \\
\hline & $\mathrm{F}$ & $595 \mathrm{Ba}$ & $800 \mathrm{Aa}$ & $656 \mathrm{Ba}$ \\
\hline & FI & $509 \mathrm{Ba}$ & 769Aa & $587 \mathrm{Ba}$ \\
\hline & I & $420 \mathrm{Cb}$ & 744ABa & $518 \mathrm{Cb}$ \\
\hline \multirow{4}{*}{15} & $\mathrm{~T}$ & $400 \mathrm{Cb}$ & $755 \mathrm{Aa}$ & $507 \mathrm{Cb}$ \\
\hline & $\mathrm{F}$ & 494Ba & $782 \mathrm{Aa}$ & $581 \mathrm{Ba}$ \\
\hline & FI & $587 \mathrm{Ba}$ & 817Aa & $656 \mathrm{Ba}$ \\
\hline & I & $342 \mathrm{Cb}$ & 743Ba & $462 \mathrm{Cb}$ \\
\hline \multirow{4}{*}{30} & $\mathrm{~T}$ & $508 \mathrm{Bb}$ & 747ABa & $580 \mathrm{Bb}$ \\
\hline & F & 759Aa & $805 \mathrm{Aa}$ & 773Aa \\
\hline & FI & 689Aa & $742 \mathrm{Ba}$ & $705 \mathrm{Aa}$ \\
\hline & I & $473 \mathrm{Cb}$ & 751Aa & $557 \mathrm{Cb}$ \\
\hline \multirow{4}{*}{45} & $\mathrm{~T}$ & $445 \mathrm{Cb}$ & $713 \mathrm{Ba}$ & $525 \mathrm{Cb}$ \\
\hline & $\mathrm{F}$ & $519 \mathrm{Ba}$ & 809Aa & $606 \mathrm{Ba}$ \\
\hline & FI & $531 \mathrm{Ba}$ & $742 \mathrm{Ba}$ & $595 \mathrm{Ba}$ \\
\hline & I & $365 \mathrm{Cb}$ & $710 \mathrm{Ba}$ & $469 \mathrm{Cb}$ \\
\hline \multirow{4}{*}{60} & $\mathrm{~T}$ & $300 \mathrm{Db}$ & $691 \mathrm{Ba}$ & $417 \mathrm{Db}$ \\
\hline & $\mathrm{F}$ & $454 \mathrm{Ba}$ & $753 \mathrm{Ba}$ & $544 \mathrm{Ba}$ \\
\hline & FI & $555 \mathrm{Ba}$ & 754Ba & $615 \mathrm{Ba}$ \\
\hline & I & $294 \mathrm{Db}$ & 707BCa & $418 \mathrm{Ca}$ \\
\hline \multirow{4}{*}{90} & $\mathrm{~T}$ & $198 \mathrm{Db}$ & 692BCa & $347 \mathrm{Da}$ \\
\hline & $\mathrm{F}$ & $218 \mathrm{Da}$ & $672 \mathrm{BCa}$ & $354 \mathrm{Da}$ \\
\hline & FI & $251 \mathrm{Da}$ & $706 \mathrm{BCa}$ & $388 \mathrm{Da}$ \\
\hline & I & $205 \mathrm{Db}$ & $696 \mathrm{BCa}$ & $353 \mathrm{Db}$ \\
\hline \multirow{4}{*}{180} & $\mathrm{~T}$ & $294 \mathrm{Db}$ & $678 \mathrm{BCa}$ & 409Da \\
\hline & $\mathrm{F}$ & $315 \mathrm{Da}$ & $594 \mathrm{Ca}$ & $399 \mathrm{Db}$ \\
\hline & FI & $346 \mathrm{Ca}$ & $725 \mathrm{Ba}$ & $460 \mathrm{Ca}$ \\
\hline & I & $178 \mathrm{Db}$ & $659 \mathrm{Ca}$ & $323 \mathrm{Db}$ \\
\hline \multicolumn{2}{|c|}{$\mathrm{CV}(\%)$} & 21,85 & 8,72 & 13,29 \\
\hline
\end{tabular}

${ }^{1}$ Dias Após o Tratamento Químico (DATQ)

${ }^{2} \mathrm{~T}=$ Testemunha; F= Fludioxonil + metalaxyl; FI= Fludioxonil+metalaxyl + Tiametoxam; $\mathrm{I}=$ Tiametoxam

*Valores seguidos pela mesma letra maiúscula, em cada coluna, não diferem entre si pelo teste de Tukey, aos 5\% de probabilidade

**Valores seguidos pela mesma letra minúscula, em cada coluna, dentro dos diferentes períodos correspondentes aos dias após o tratamento químico, não diferem entre si pelo teste de Tukey, aos $5 \%$ de probabilidade

Os benefícios do tratamento das sementes com fungicida na preservação da germinação das sementes foram observados em todos os períodos de armazenamento, com exceção para os períodos de 0 e 45 dias após o tratamento químico, nos quais não houve diferença em relação à testemunha, embora os valores foram 
numericamente maiores para as sementes tratadas (Tabela 2). Por outro lado, as sementes tratadas com a mistura fungicida + inseticida, apresentaram melhor germinação em relação à testemunha apenas aos 30,60 e 180 dias após o armazenamento. Ressalta-se, entretanto, que, em alguns casos, as sementes tratadas com fungicida apresentaram maior germinação e vigor quando comparadas com as sementes tratadas com fungicida + inseticida, conforme pode ser observado nos períodos de 15 e 90 dias após o tratamento químico para o teste de germinação, nos períodos de 30 e 45 dias após o tratamento químico para o teste de envelhecimento acelerado e a partir de 60 dias após o tratamento químico para o teste de emergência de plântulas em campo. Observando os resultados obtidos no teste de sanidade (Tabela 3), verifica-se que, de maneira geral, para os períodos de 90 e 180 dias após o tratamento químico, houve alta incidência de fungos Aspergillus spp. e Rhizopus spp. nas sementes submetidas ao tratamento fungicida + inseticida, quando comparado com o tratamento que recebeu apenas o fungicida, explicando, em parte, os piores resultados de germinação e de vigor apresentados na Tabela 2.

Para o teste de condutividade elétrica (Tabela 2), não foi observado o mesmo comportamento em separar os diferentes tratamentos químicos aplicados às sementes. Cabe ressaltar que os produtos utilizados no tratamento das sementes parecem não ter influenciado o teste de condutividade elétrica, assegurando a confiabilidade dos resultados, conforme observado também em pesquisa desenvolvida por Marchi e Cicero (2003) com sementes de milho. Observa-se na Tabela 2, que as maiores leituras foram verificadas para sementes armazenadas em períodos superiores a 30 dias após o tratamento químico, o que pode ser resultado da perda do potencial fisiológico durante o armazenamento, uma vez que membranas mal estruturadas e células danificadas estão, geralmente, associadas com o processo de deterioração da semente, afetando o vigor (Powell e Matthews, 1981).

O tratamento das sementes com fungicida e com fungicida + inseticida, dentro de cada época de avaliação, foi eficiente no controle de fungos de armazenamento quando comparados com os demais tratamentos (Tabela 3). Porém, analisando o efeito do tratamento fungicida + inseticida ao longo do armazenamento, observa-se que houve aumento significativo na incidência do fungo Aspergillus spp. a partir 90 dias após o tratamento químico. Assim, pode-se afirmar que o melhor potencial fisiológico das sementes até 60 dias após o tratamento químico, evidenciado nos testes de germinação, de envelhecimento acelerado e de emergência de plântulas em campo (Tabela 2), para os tratamentos fungicida e fungicida + inseticida, deveu-se, em parte, ao controle eficiente dos fungos Aspergillus spp. e Rhizopus spp.. Cabe ressaltar também que a baixa germinação e o baixo vigor das sementes tratadas com o inseticida podem estar relacionados com a deterioração durante o armazenamento, bem como, com o efeito fitotóxico do produto inseticida, quer aplicado isoladamente ou em conjunto com o fungicida, corroborando com a pesquisa de Scarpellini e Nakamura (2002), que com o objetivo de estudar o efeito do tratamento de sementes com o inseticida thiamethoxam (Cruiser 700 WS) no controle do tripes Enneothrips flavens na cultura do amendoim, cv. Tatu constataram efeitos fitotóxicos durante o armazenamento das sementes. Da mesma maneira, Gotardo et al. (2001), estudando o inseticida thiamethoxam em sementes de milho, concluíram que ouso do inseticida fica condicionado ao período de armazenamento das sementes, devendo ser aplicado somente no momento da semeadura, para que não ocorra fitotoxicidade. Também, Bittencourt et al. (2000), estudando os inseticidas carbofuran (Furazin 310TS), thiodicarb (Semevin 350RA), thiodicarb + molibdênio e boro (Futur 300) e thiamethoxan (Cruiser 70WS) na germinação e vigor de sementes de milho com diferentes períodos de armazenamento, verificaram que todos os inseticidas causaram reduções significativas na germinação e no vigor das sementes, avaliado pelos testes de frio e de condutividade elétrica, com intensidade variável em função do tipo de híbrido, do produto e do período de armazenamento.

Por outro lado, para os índices de crescimento e de vigor de plântulas obtidos por meio da análise computadorizada (Tabela 4), o tratamento fungicida não diferiu do tratamento fungicida + inseticida, em todo período de armazenamento, com exceção para 180 dias de armazenamento para o índice de vigor, que acusou melhor desempenho para as sementes tratadas com a mistura dos produtos

Embora não tenha sido constatada a mesma sensibilidade na separação dos tratamentos fungicida e fungicida + inseticida, por meio do programa computacional, como foi observado nos testes de germinação, envelhecimento acelerado e emergência de plântulas em campo (Tabela 2), a análise automatizada possibilitou a separação desses tratamentos em relação à testemunha e ao tratamento apenas com inseticida. Já, 
com relação ao índice de uniformidade de crescimento de plântulas, não foi observada eficiência para diferenciar os tratamentos (Tabela 4). Para períodos de armazenamento superiores a trinta dias, tanto para IC, quanto para IV, observaram-se valores superiores às épocas de armazenamento precedentes o que pode indicar a ocorrência esporádica de menor número de sementes mortas plântulas anormais, as quais são eliminadas do teste.

Observando os resultados apresentados na Tabela 4, de maneira geral, os maiores valores dos índices de crescimento, de uniformidade e de vigor foram obtidos para sementes armazenadas até 60 dias após o tratamento químico, sendo que, a partir deste período de armazenamento os valores decresceram significativamente. Da mesma maneira, analisando os resultados do teste de envelhecimento acelerado e de emergência de plântulas em campo (Tabela 2), a queda significativa do vigor ocorreu a partir dos 60 dias após o tratamento químico.

Além de apresentar maior rapidez na obtenção dos resultados, a análise computadorizada mostrou de forma mais evidente a redução do potencial fisiológico no decorrer dos períodos de armazenamento (Tabela 4), quando comparados aos testes de envelhecimento acelerado e de emergência de plântulas em campo (Tabela 2), ou seja, a redução do potencial fisiológico foi detectada antecipadamente pela análise computadorizada de plântulas. A eficiência do método de análise computadorizada e sua vantagem em relação a outros métodos na avaliação do potencial fisiológico de sementes também foram constatadas por outros pesquisadores, como, por exemplo, Sekharan (2005), que trabalhando com lotes de sementes de soja envelhecidas artificialmente, com germinação variando entre $83 \%$ e $95 \%$, constatou a eficiência das análises feitas pelo programa computacional na detecção de diferenças no potencial fisiológico, especialmente quando as variações entre lotes eram mais acentuadas. Marcos Filho et al. (2006), trabalhando com sementes de melão, concluíram que os índices obtidos pela análise computadorizada de plântulas se relacionavam com os resultados obtidos pelos testes de vigor rotineiramente utilizados para a avaliação da qualidade das sementes, sendo uma alternativa rápida e objetiva para a avaliação do vigor das sementes. Também, Marcos Filho et al. (2009), trabalhando com sementes de soja, verificaram que o vigor das sementes, avaliado por meio deste programa, apresentou valores consistentes em comparação com os resultados de primeira contagem e de velocidade de germinação, de envelhecimento acelerado e de emergência de plântulas. Por sua vez, Gomes Junior et al. (2009) verificaram eficiência do programa computacional na avaliação dos efeitos do condicionamento fisiológico de sementes de milho doce. Assim, com base nos resultados observados nesta pesquisa, a análise computadorizada demonstrou ser uma importante técnica para avaliação do potencial fisiológico de sementes de amendoim.

\section{CONCLUSÃO}

A análise computadorizada de plântulas apresenta eficiência equivalente aos testes de envelhecimento acelerado e de emergência de plântulas em campo na identificação de diferenças entre tratamentos com ausência ou presença de fungicida e de fungicida associado à inseticida nas sementes de amendoim.

\section{REFERÊNCIAS}

ANGELINI, A.C.; MARTINS, L. Avaliação de diversas formulações disponíveis para a proteção do tegumento e aplicação de corante em sementes de amendoim. Informações Técnicas do Serviço de Controle de Qualidade, D.S.M.M. -CATI, 1986, 5p.

ASSOCIATION OF OFFICIAL SEED ANALYSTIS. Seed vigor testing handbook. Lincoln, 1983, 93p.

BACKMAN, P.A.; HAMMOND, J.M. Germination losses associated with delayed application of seed treatment fungicides after peanut shelling. Plant Disease Reporter, n.60, p.1-2, 1976.

BITTENCOURT, S.R.M.;FERNANDEZ,M.A.; RIBEIRO, M.C.; VIEIRA, R.D. Desempenho de sementes de milho tratadas com inseticidas sistêmicos. Revista Brasileira de Sementes. v.22, n.2, p.86-93, 2000.

BRASIL. Ministério da Agricultura e Reforma Agrária. Secretaria Nacional de Defesa Agropecuária. Departamento Nacional de Produção Vegetal. Coordenação de Laboratório Vegetal. Regras para análise de sementes. Brasília, DF, 1992. 365p.

DELOUCHE, J.C.; BASKIN, C.C. Accelerated aging techniques for predicting the relative storability of seed lots. Seed Science and Technology, v.1, p.427-452, 1973.

DELOUCHE, J.C. Observaciones sobre deterioracion de semillas. Semillas, v.1, n.1, p.8-11, 1986. 
FADIGAS JUNIOR, M.; SUPLICY FILHO, N. Inseticidas sistêmicos em sementes de amendoim, visando ao controle do tripes. O Biológico, v.27, n.8, p.187, 1961.

GELMOND, H.G. Growth and development of the peanut plant (Arachis hypogea) in relation to seedling evaluation in the germination test. Proceedings of the International Seed Testing Association, v.1, n.36, p.121-131, 1971.

GOMES JUNIOR, F.G.; CHAMMA, H.M.C.P. Eficiência de um sistema computadorizado de processamento de imagens para avaliação do vigor de sementes de feijão. In: CONGRESSO NACIONAL DE PESQUISA DE FEIJÃO, 9., 2008, Campinas. Anais... Instituto Agronômico de Campinas, 2008. p.685-688.

GOMES JUNIOR, F.G.; MONDO, V.H.V.; CICERO, S.M.; McDONALD, M.B.; BENNETT, M.A. Evaluation of priming effects on sweet corn seeds by SVIS. Seed Technology, v.31, n.1, p.95-100, 2009.

GOTARDO, M.; BITTENCOURT, S.R.M.; PEREIRA, L.M.A.; VIEIRA, R.D.; GOTARDO JUNIOR, J.R. Qualidade fisiológica de milho tratadas com diferentes inseticidas. Revista Ceres, v.48, n.278, p.511-516, 2001.

HOFFMASTER, A.L.; FUJIMURA, K.; McDONALD, M.B.; BENETT, M.A. An automated system for vigour testing three-day-old soybean seedling. Seed Science and Technology, n.1, v.33, p.701-713, 2003.

HOFFMASTER, A.L.; XU, L.; FUJIMURA, K.; McDONALD, M.B.; BENNETT, M.A.; EVANS, A.F. The Ohio State University Seed Vigor Imaging System (SVIS) for soybean and corn seedlings. Seed Technology, v.27, n.1, p.7-26, 2005.

KRZYZANOWSKI, F.C.; MIRANDA, Z.F.S. Relatório do Comitê de Vigor da ABRATES. Informativo ABRATES, v.1, n.1, p.7-25, 1990.

LAGO, A.A.; TOLEDO, F.F. Tratamento de sementes de amendoim com inseticidas sistêmicos combinados a fungicida. In: SEMINÁRIO BRASILEIRO DE SEMENTES, 2., 1968. Anais... 1970, p.193-198.

LAGO, A.A.; ORTOLANI, D.B.;ZINK, E.; FERNANDES, C.O. Efeitos de diversos tratamentos fungicidas na longevidade de sementes de amendoim. Semente, v.2, n.2, p.26-31, 1976.

LUCA FILHO, O.A. Metodologia dos testes de sanidade em sementes. In: SOAVE, J.; WETZEL, M.M.V.S.(Ed.). Patologia de Sementes, Fundação Cargill, 1987, p.276298.

MACHADO, J.C. Introdução à patologia de sementes.
In: SOAVE, J.; WETZEL, M.M.V.S. (Ed.). Patologia de Sementes, Fundação Cargill, 1987, p.3-17.

MAEDA, J.A.; LAGO, A.A.; GERIN, M.A.N. Tratamentos com fungicidas no comportamento de sementes de amendoim. Bragantia, v.1, n.54, p.103-111, 1995.

MARCHI, J.L.; CICERO, S.M. Influence of chemical treatment of maize seeds with different levels of mechanical damage on electrical conductivity values. Seed Science and Technology, v.31, n.1, p.481-486, 2003.

MARCOS FILHO, J.; CICERO, S.M.; SILVA, W.R. Avaliação da qualidade das sementes. Piracicaba: FEALQ, 1987, 230p.

MARCOS FILHO, J.; BENNETT, M.A.; EVANS, A.S.; GRASSBAUGH, E.M. Assessment of melon seed vigour by an automated computer imaging system compared to traditional procedures. Seed Science and Technology, v.34, n.2, p.507-519, 2006.

MARCOS FILHO, J.; KIKUTI, A.L.P.; LIMA, L.B. Métodos para avaliação do vigor de sementes de soja, incluindo a análise computadorizada de imagens. Revista Brasileira de Sementes, v.31, n.1, p.102-112, 2009.

MENTEN, J.O.M. Importancia do tratamento de sementes. In: MENTEN, J.O.M., (Ed.) Patógenos em sementes: detecção, danos e controle químico. In: SEMANA DE ATUALIZAÇÃO EM PATOLOGIA DE SEMENTES, 2., Piracicaba, 1991. Anais... Piracicaba, FEALQ, 1991. p.203-224.

MORAES, S.A. Teste de sanidade de sementes de amendoim. In: SOAVE, J.; WETZEL, M.M.V.S. (Ed.). Patologia de Sementes. Campinas: Fundação Cargill, 1987, p.347-357.

OTONI, R.R.; McDONALD, M.B. Moisture and temperature effects on maize and soybean seedlings using the seed vigor imaging system. Seed Technology, v.27, n.2, p.243-247, 2005.

PANDEY, S.; SINGH, D.K. Soil dehydrogenase, phosphomonoesterase and arginine deaminase activities in an insecticide treated groundnut (Arachis hypogaea L.). Chemosphere, v.63, n.1, p.869-880, 2006.

POWELL, A.A.; MATTHEWS, S. Evaluation of controlled deterioration: a new vigour test for small seeds vegetables. Seed Science and Technology, v.9, n.2, p.633-640, 1981.

PRETE, C.E.C.; CICERO, S.M. Escolha manual, seleção eletrônica pela cor, tratamento fungicida e qualidade de sementes de amendoim. Anais da Escola Superior de Agricultura, v.44, p.37-56, 1987. 
RANDALL-SCHADEL, B.L.; BAILEY, J.E.; BEUTE, M.K. Seed transmission of Cylindrocladium parasiticum in peanut. Plant Disease, v.85, n.4. p.362-370, 2001.

SADER, R.; CHALITA, C.; TEIXEIRA, L.G. Influência do tamanho e do beneficiamento na injúria mecânica de sementes de amendoim. Revista Brasileira de Sementes, v.13, n.1, p.45-51, 1991.

SAKO, Y.; McDONALD, M.B.; FUJIMURA, K.; EVANS, A.F.; BENETT, M.A. A system for automated seed vigour assessment. Seed science and technology. v.29, n.2, p.625636, 2001.

SCARPELLINI, J.R.; NAKAMURA, G. Controle do tripes Enneothrips flavens (moulton, 1941) (thysanoptera: thripidae) e efeito na produtividade do amendoim. Arquivo do Instituto Biológico de São Paulo, v.69, n.3, p.85-88, 2002.

SEKHARAN, S. Aged soybean seeds - their physiology and vigor assessment. 2006. 180f. Tese (Ph.D). The Ohio State University, Columbus/OH/EUA, 2005.
TEIXEIRA, E.F.; CICERO, S.M.; DOURADO NETO, D. Noções básicas sobre imagens digitais: captura, processamento e reconhecimento voltados para a pesquisa em tecnologia de sementes. Informativo ABRATES, v.13, n. 1, p.59-65, 2003.

TELLA, R.; LAGO, A.A.; ZINK, E. Efeito de diversos níveis de umidade e tratamento fungicida na longevidade de sementes de amendoim. Bragantia, v.1, n.35, p.335$342,1976$.

TOSELLO, J.; ORTOLANI, D.B.; MASCHIETTO, J.C. Observações sobre a conservação de sementes de amendoim. In: SEMINÁRIO BRASILEIRO DE SEMENTES, 2., Pelotas, 1968, Anais... Rio de Janeiro, 1970, p.333-338.

USBERTI, R. Efeitos da época de aplicação de fungicida, tamanho e origem de sementes na germinação e potencial de armazenamento de amendoim (Arachis hypogea, L.). In: CONGRESSO BRASILEIRO DE SEMENTES, 3., 1983. Campinas. Resumos... Brasília: Associação Brasileira de Sementes, 1983. p.189. 